\title{
Non abelian Bianchi identities, monopoles and gauge invariance
}

\author{
Claudio Bonati* \\ Dipartimento di Fisica, Università di Pisa and INFN, Largo Pontecorvo 3, I-56127 Pisa, Italy. \\ E-mail: claudio.bonatiepi.infn.it \\ Massimo D'Elia \\ Dipartimento di Fisica, Università di Genova and INFN, Via Dodecaneso 33, 16146 Genova, \\ Italy. \\ E-mail: massimo.delia@ge.infn.it
}

\section{Adriano Di Giacomo}

Dipartimento di Fisica, Università di Pisa and INFN, Largo Pontecorvo 3, I-56127 Pisa, Italy. E-mail: digiaco@df.unipi.it

\section{Luca Lepori}

Scuola Internazionale Superiore di Studi Avanzati and INFN, Via Beirut 2-4, 34151 Trieste, Italy. E-mail: leporiesissa.it

\section{Fabrizio Pucci}

Dipartimento di Fisica, Università di Firenze and INFN, Via Sansone 1, 50019 Sesto Fiorentino, Firenze, Italy. ${ }^{\dagger}$

E-mail: pucciephysik.uni-bielefeld.de

\begin{abstract}
A direct connection is proved between the Non-Abelian Bianchi Identities and the Abelian Bianchi identities for the 't Hooft tensor in a generic gauge; the existence of a magnetic current is related to the violation of NABI's. Using this relation it is shown that not all gauges are equivalent to detect monopoles on the lattice, that e.g. the Maximal Abelian Gauge is a legitimate choice while the Landau gauge is not. Nevertheless monopole condensation is found to be a gauge invariant property.
\end{abstract}

The XXVIII International Symposium on Lattice Filed Theory

June 14-19,2010

Villasimius, Sardinia Italy

\footnotetext{
${ }^{*}$ Speaker.

†Present address: Fakultät für Physik, Universität Bielefeld, D-33615 Bielefeld, Germanyy.
} 


\section{Introduction}

Monopoles have been proposed to be the excitations of QCD responsible for the confinement of color, which, in this picture, is interpreted as a consequence of the dual superconductivity of the vacuum induced by the monopole condensation [1,2].

Because of this proposal, a big activity has developed during the years in the lattice community to detect and study monopoles in numerically generated QCD configurations. These studies can be divided into two main groups with different working strategies:

- the study of the (dual) symmetry of the vacuum state

- the direct observation of monopoles in lattice configurations

In the studies of the first group an order parameter for dual superconductivity is introduced: this is defined as the vacuum expectation value (vev) of a magnetically charged operator $\mu$. $\langle\mu\rangle$ is zero if the magnetic charge is superselected and nonzero in the dual superconducting phase (see e.g. $[3,4,5,6,7,8]$ and references therein). The works of the second group try instead to extract a monopole effective action or to check monopole dominance by direct detection of monopoles in lattice configurations (see e.g. $[9,10,11]$ and references therein).

In this paper we shall analyze some general properties of monopoles in non abelian gauge theories, whose applications are more direct in the studies of the second group but that are also interesting from the point of view of the works of the first one.

The standard method to detect monopoles in lattice configurations was first introduced in Ref. [12] for the $U(1)$ gauge theory: a Dirac string is identified by any excess over $2 \pi$ of the abelian phase of a plaquette and a monopole is located inside an elementary cube whenever a net number of Dirac strings crosses its boundary plaquettes. This procedure is well defined since in $U(1)$ gauge theory the abelian phase of a plaquette is gauge invariant. For non abelian gauge theories this recipe must be modified: one has first to fix a gauge, and then to apply the above prescription to the components of the non abelian field directed along an abelian subgroup of the original gauge group (abelian projection).

While for the 't Hooft-Polyakov monopole of Ref. $[13,14]$ the magnetic $U(1)$ group is naturally identified with the little group of the Higgs vev in the unitary representation, in gauge theories without Higgs field there is no obvious preferred gauge direction. Indeed it was proposed in Ref. [15] that any operator in the adjoint representation of the group could be used as an effective Higgs field to identify the magnetic $U(1)$, physics being for some reason independent of that choice. It was however observed in numerical simulations that the number and the position of the monopoles detected in a given configuration was strongly dependent on the abelian projection.

For the interpretation of the color confinement as dual superconductivity it is crucial to be able to define monopoles in a gauge invariant way. We will show that this is possible by using the Non Abelian Bianchi Identities (NABI) and that for any magnetically charged gauge field configuration a preferred direction in color space exists, which identifies the correct magnetic $U(1)$ subgroup. As a consequence monopoles are gauge invariant objects but their detection by the recipe of Ref. [12] it is not; this techniques works well for a class of gauges which include the maximal abelian gauge but it fails for the Landau gauge, where no monopoles are detected. Dual superconductivity, defined as the breaking of the magnetic $U(1)$, is also gauge invariant. 


\section{Abelian and Non-Abelian Bianchi Identities}

Abelian Bianchi identities are $\partial_{\mu} F_{\mu \nu}^{*}=0$, where $F_{\mu \nu}^{*}=\frac{1}{2} \varepsilon_{\mu v \rho \sigma} F_{\rho \sigma}$ is the dual of the abelian field strength, and their violation naturally defines the magnetic current

$$
j_{v}=\partial_{\mu} F_{\mu v}^{*}
$$

which is conserved because of the antisymmetry of the $F_{\mu v}^{*}$ tensor: $\partial_{v} j_{v}=0$. The Non Abelian Bianchi Identities are the covariant generalization of the abelian ones for non abelian gauge theories and their violation defines the current

$$
J_{v}=D_{\mu} G_{\mu v}^{*}
$$

which is not difficult to show to be covariantly conserved: $D_{v} J_{v}=0$.

In non abelian gauge theories the abelian field strength $F_{\mu \nu}$ is defined by means of the 't Hooft tensor, which reduces to the abelian field strength of the residual $U(1)$ in the unitary gauge; its introduction goes back to Ref. [13] for the group $S U(2)$, while the generalization for an arbitrary compact gauge group was developed in Ref. [16].

Because of the Coleman-Mandula theorem, the four components of the current $J_{v}$ defined in Eq. (2.2) commute with each other; to expose the gauge invariant content of this equation is thus possible to simultaneously diagonalize all its component. A convenient basis for the diagonal matrices is that of the fundamental weights $\phi_{0}^{a}, a=1, \ldots, r$, where $r$ is the rank of the gauge group. A fundamental weight $\phi_{0}^{a}$ is associated to each of the simple roots $\vec{\alpha}^{a}$ of the group algebra; its commutations rules with the elements of the Cartan base are $\left[\phi_{0}^{a}, H_{i}\right]=0$ and $\left[\phi_{0}^{a}, E_{ \pm \vec{\alpha}}\right]= \pm\left(\vec{c}^{a}\right.$. $\vec{\alpha}) E_{ \pm \vec{\alpha}}$ and for the simple roots $\vec{c}^{a} \cdot \vec{\alpha}^{b}=\delta^{a b}$. Let $\phi_{I}^{a}$ be the adjoint representation operator equal to $\phi_{0}^{a}$ in the gauge that diagonalize $J_{v}$; then the gauge invariant content of Eq. (2.2) is

$$
\operatorname{Tr}\left(\phi_{I}^{a} D_{\mu} G_{\mu v}^{*}\right)=\operatorname{Tr}\left(\phi_{I}^{a} J_{v}\right)
$$

Let us now denote by $\phi^{a}$ the matrix which is equal to $V(x) \phi_{0}^{a} V(x)^{\dagger}$ in the gauge in which $J_{V}$ is diagonal, $V(x)$ being a generic gauge transformation. It was proved in Ref. [17] that, for a generic compact gauge group, the abelian magnetic current $j_{v}^{a}$ in the abelian projection defined by the operator $\phi^{a}$ satisfies

$$
\partial_{\mu} F_{\mu \nu}^{a *}=j_{v}^{a}=\operatorname{Tr}\left(\phi^{a} J_{v}\right)
$$

where $F_{\mu \nu}^{a *}$ is the 't Hooft tensor in the given abelian projection. This equation shows that the existence of a magnetic current is related to the violations of the NABI's and that its value is just the abelian projection of that violation, thus revealing the hidden gauge invariance of the monopole's definition.

\section{The 't Hooft-Polyakov monopole}

In this section we will check the equation Eq. (2.4) for the soliton solution of Ref. [13, 14] and we will make some simple observations whose relevance will become clear in the next sections.

The solution of Ref. [13, 14] can be written in the form

$$
\phi^{a}(\vec{r})=H(r) \frac{r^{a}}{r} \quad A_{0}=0 \quad A_{i}^{a}=\varepsilon_{i a k} \frac{r^{k}}{g r^{2}}[1-K(r)]
$$


where $H, K$ are two functions whose specific form depends on the details of the Higgs potential. Their universal features are their asymptotics: $\lim _{r \rightarrow 0} H(r)=0$ and $1-K(x) \propto x^{2}$ for small $x$ in order to ensure regularity; $\lim _{r \rightarrow \infty} H(r)=$ const and $\lim _{x \rightarrow \infty} K(x)=0$ to have a topologically stable solution of finite total energy.

The gauge in which the solution Eq. (3.1) is written is usually called the "hedgehog" one and it is trivial to verify that it satisfies the Landau gauge condition $\partial_{\mu} A_{\mu}=0$. The asymptotic abelian magnetic field at large distance in this gauge can be shown to be given by the expression

$$
b^{i}(\vec{r}) \approx \frac{r^{i}}{g r^{2}} \frac{z}{r}
$$

and thus the magnetic charge $Q_{m}$, calculated by the flux at infinity, is zero.

Let us now consider the unitary gauge, that is the gauge in which the Higgs field is rotated into a fixed direction in color space. The explicit form of the gauge field can be computed starting from Eq. (3.1) (see e.g. Ref. [18]) and it is simple to verify that the solution in the unitary gauge satisfies the equation

$$
\partial_{\mu} A_{\mu}^{ \pm}+i g\left[A_{\mu}^{3}, A_{\mu}^{ \pm}\right]=0
$$

which is the continuum form of the maximal abelian gauge (MAG) introduced in Ref. [19]. In this gauge it is easy to show that the temporal component (the only non-vanishing one) of the non abelian current Eq. (2.2) is given by

$$
J_{0}=D_{i} B_{i}=\frac{2 \pi}{g} \delta^{3}(\vec{r}) \sigma_{3}
$$

which is diagonal in color space: the unitary gauge coincides with the abelian projection indicated by $\phi_{I}^{a}$ in the previous section. For $S U(2)$ there is only one fundamental weight, $\phi_{0}=\frac{1}{2} \sigma_{3}$, associated to the +1 simple root. By direct calculation it can also be shown that the temporal component of the abelian magnetic current is equal to

$$
j_{0}=\vec{\nabla} \cdot \vec{b}=\frac{2 \pi}{g} \delta^{3}(\vec{r})
$$

consistently with the theorem of the previous section, Eq. (2.4). In the unitary gauge the magnetic charge is thus $Q_{m}=\frac{1}{2 g}$; since the elementary electric charge is given by $Q_{e}=\frac{g}{2}, Q_{m}$ is equal to two Dirac units.

To summarize we have shown that monopoles are related to NABI's violations and they are thus gauge invariant objects; however, because of the presence of a preferred direction in color space (that of the NABI's violation), the magnetic charge computed by using the flux at infinity depends on the gauge choice. In particular we have just shown that, for the 't Hooft-Polyakov soliton, the magnetic charge in the Landau gauge is zero, while in the maximal abelian gauge it is equal to two Dirac units.

\section{General monopole configuration}

The conclusions of the previous section can be generalized to an arbitrary configuration by using the following theorem of Ref. [20]: the magnetic monopole term in the multipole expansion 
of a generic static field configuration is abelian (i.e. satisfies abelian equations of motion) and can be gauged along a fixed direction in color space.

Up to a global gauge transformation we can suppose the asymptotic non abelian magnetic field of the configuration $A_{\mu}$ to be directed along the 3-axis in color space; at large $r$ it is then fixed by the total magnetic charge $m$ (in Dirac units) to be

$$
\vec{B}=\frac{m}{2} \frac{\vec{r}}{2 g r^{3}} \sigma_{3}
$$

By adding and subtracting to the gauge field a term $\tilde{A}_{\mu}$ given by the 't Hooft-Polyakov solution in the unitary gauge, multiplied by $\frac{m}{2}$, we can see the field $A_{\mu}$ as a superposition of a 't HooftPoliakov-like term $\tilde{A}_{\mu}$ and a topologically trivial term $\tilde{A}_{\mu}^{(0)}=A_{\mu}-\tilde{A}_{\mu}$. Since the 't Hooft tensor is linear in the gauge field, the asymptotic abelian magnetic field of $\tilde{A}_{\mu}^{(0)}$ is zero, and the results of the previous section can extended to a general static configuration: in the MAG, or in any gauge which differs from it by an asymptotically trivial gauge transformation, the flux of the abelian magnetic field at large distance gives the correct magnetic charge. In other gauges the flux at infinity will be generally smaller than the MAG one and for the Landau gauge it will be vanishing.

For a non static configuration, the same argument can be applied to the superposition of the given configuration and its time-reversed one, in order to isolate the asymptotic magnetic field from the electric field.

\section{Monopole condensation}

From equation Eq. (2.4) it follows that in $S U(2)$ gauge theory the magnetic charge in the MAG is given by

$$
Q_{I}=\int \mathrm{d}^{3} x \operatorname{Tr}\left(\phi_{I} J_{0}(x)\right)
$$

A magnetically charged operator $O(x)$ satisfies the commutation rule

$$
\left[Q_{I}, O(x)\right]=m O(x) \quad m \neq 0
$$

and the magnetic $U(1)$ symmetry is broken if a charged operator exists for which $\langle O\rangle \neq 0$. We will show now that this property is gauge invariant: in a generic abelian projection $V$ the magnetic charge $Q_{V}$ has the form in Eq. (5.1) with $\phi_{I} \rightarrow V(x) \phi_{I} V(x)^{\dagger}$ and, since the integrand is gauge invariant, we can compute the trace in the gauge in which $J_{0}$ and $\phi_{I}$ are diagonal. Since $V(x) \phi_{I} V(x)^{\dagger}$ is an element of the group algebra it can be expanded in the Cartan base:

$$
V(x) \phi V(x)^{\dagger}=C(x, V) \phi_{I}+\sum_{\vec{\alpha}} E_{\vec{\alpha}} D^{\vec{\alpha}}(x, V)
$$

In the chosen gauge only the first term contributes and thus

$$
\left[Q_{I}, O(x)\right]=m C(x, V) O(x)
$$

Since $C(x, V)$ is generically non-vanishing, the operator $O$ will be charged also with respect to $Q_{V}$ and the corresponding $U(1)$ magnetic symmetry will be broken. For a general gauge group the only difference is that in Eq. (5.3) the term $C(x, V) \phi_{I}$ has to be replaced by a sum over the fundamental weights $\phi_{I}^{a}(a=1, \ldots, r)$. 


\section{Monopoles detection on the lattice}

The detection of monopoles in lattice configurations is performed by measuring the abelian magnetic flux, in a given abelian projection, through the surface of elementary cubes [12]. The previous analysis shows that the magnetic flux depends on the chosen abelian projection: the "correct" magnetic charge is measured in the MAG, while in other gauges it is expected to be smaller.

A direct test of the previous picture is presented in Ref. [21]. The starting point is a configuration in the MAG; in this gauge it is known that the flux on the boundary of an elementary cube gives a reliable estimate of the flux at infinity (see Ref. [22]), so we can safely apply the recipe of Ref. [12] to locate monopoles. If we now assume that the monopole is at the center of a cube and the Dirac string along the $z$ direction, we can perform the gauge transformation

$$
U(a)=\exp \left(-i \phi \frac{\sigma_{3}}{2}\right) \exp \left(-i a \theta \frac{\sigma_{2}}{2}\right) \exp \left(i \phi \frac{\sigma_{3}}{2}\right)
$$

where $\theta, \phi$ are the polar angles and $0 \leq a \leq 1$ is a free parameter. For $a=0$ one stays in the MAG, for $a=1$ the transformation in Eq. (6.1) is the unitary matrix bringing from the MAG to the Landau gauge (see e.g. [18]). In the gauge defined by the parameter $a$, the magnetic charge, defined by the abelian magnetic flux at infinity, is given by

$$
\frac{Q(a)}{Q(0)}=\frac{1+\cos (a \pi)}{2}
$$

The comparison between Eq. (6.2) and the lattice measurements was performed in Ref. [21]. Although in its derivation we completely neglected all sort of discretization errors, Eq. (6.2) qualitatively well describes the observed behaviour of $Q(a)$.

\section{Conclusions}

We have shown that monopoles are gauge invariant objects and that each configuration with non-zero magnetic field selects a natural direction in color space, induced by the violations of the non-abelian Bianchi identities. This direction is correctly identified by the maximal abelian gauge, which is the gauge to be used to detect monopoles in lattice configuration by the DeGrandToussaint recipe. While monopole condensation is a gauge invariant fact, monopole detection is strongly affected by the gauge choice in a well understood way.

\section{References}

[1] S. Mandelstam, Vortices and quark confinement in non-Abelian gauge theories. Phys. Rep. 23, 245 (1976).

[2] G. 't Hooft, Gauge theories for strong interactions, in A. Zichichi (ed.) New phenomena in Subnuclear Physics, Plenum Press, New York (1977).

[3] A. Di Giacomo, Mechanisms of colour confinement. Acta Phys. Polon. B 25215 (1994).

[4] L. Del Debbio, A. Di Giacomo, G. Paffuti, P. Pieri, Colour confinement as dual Meissner effect: SU (2) gauge theory. Phys. Lett. B 355, 255 (1995) [arXiv: hep-lat/9505014]. 
[5] A. I. Veselov, M. I. Polikarpov, M. N. Chernodub, Monopole order parameter in SU(2) lattice gauge theory. JETP Lett. 63, 411 (1996).

[6] A. Di Giacomo, B. Lucini, L. Montesi, G. Paffuti, Color confinement and dual superconductivity of the vacuum. I. Phys. Rev. D 61, 034503 (2000) [arXiv: hep-lat/9906024].

[7] P. Cea, L. Cosmai, Gauge invariant study of the monopole condensation in non-Abelian lattice gauge theories. Phys. Rev. D 62, 094510 (2000) [arXiv: hep-lat/ 0006007 ].

[8] J. M. Carmona, M. D’Elia, A. Di Giacomo, B. Lucini, G. Paffuti, Color confinement and dual superconductivity of the vacuum. III. Phys. Rev. D 64, 114507 (2001)

[arXiv:hep-lat/0103005].

[9] T. Suzuki, I. Yotsuyanagi, Possible evidence for Abelian dominance in quark confinement. Phys. Rev. D 42, 4257 (1990).

[10] M. I. Polikarpov, Recent Results on the Abelian Projection of Lattice Gluodynamics. Nucl. Phys. B (Proc. Suppl.) 53, 134 (1997) [arXiv: hep-lat/9609020].

[11] T. Suzuki, M. Hasegawa, K. Ishiguro, Y. Koma, T. Sekido, Gauge invariance of color confinement due to the dual Meissner effect caused by Abelian monopoles. Phys. Rev. D 80, 054504 (2009) [arXiv:0907.0583 [hep-lat]].

[12] T. A. DeGrand, D. Toussaint, Topological excitations and Monte Carlo simulation of Abelian gauge theory. Phys. Rev. D 22, 2478 (1980).

[13] G. 't Hooft, Magnetic monopoles in unified gauge theories. Nucl. Phys. B 79, 276 (1974).

[14] A. M. Polyakov, Particle spectrum in quantum field theory. JETP Lett. 20, 194 (1974).

[15] G. 't Hooft, Topology of the gauge condition and new confinement phases in non-abelian gauge theories. Nucl. Phys. B 190, 455 (1981).

[16] A. Di Giacomo, L. Lepori and F. Pucci, Homotopy, monopoles and 't Hooft tensor in QCD with generic gauge group. JHEP 0810, 096 (2008) [arXiv : 0810.4226 [hep-lat]].

[17] C. Bonati, A. Di Giacomo, L. Lepori and F. Pucci, Monopoles, abelian projection, and gauge invariance. Phys. Rev. D 81, 085022 (2010) [arXiv: 1002 . 3874 [hep-lat]].

[18] Y. M. Shnir Magnetic Monopoles. Springer (2005).

[19] A. S. Kronfeld, M. L. Laursen, G. Schierholz, U. J. Wiese, Monopole Condensation and Color Confinement. Phys. Lett. B 198, 516 (1987).

[20] S. Coleman, The Magnetic Monopole Fifty Years Later, in A. Zichichi (ed.) The unity of the fundamental interactions, Plenum Press, New York (1983).

[21] C. Bonati, A. Di Giacomo, M. D’Elia, Detecting monopoles on the lattice. [arXiv: 1009.2425 [hep-lat]], submitted for publication.

[22] L. Del Debbio, A. Di Giacomo, M. Maggiore, S. Olejnik, Confinement and monopoles in lattice QCD. Phys. Lett. B 267, 254 (1991). 